\title{
The Unwounded Skin Remodeling in Animal Models of Diabetes Types 1 and 2
}

\author{
M. KNAŚ ${ }^{1}$, M. NICZYPORUK ${ }^{2}$, A. ZALEWSKA ${ }^{3}$, H. CAR ${ }^{4}$ \\ ${ }^{1}$ Research Laboratory of Cosmetology, Medical University, Bialystok, Poland, ${ }^{2}$ Research \\ Laboratory of Esthetic Medicine, Medical University, Bialystok, Poland, ${ }^{3}$ Department of \\ Pedodontics, Medical University, Bialystok, Poland, ${ }^{4}$ Department of Experimental Pharmacology \\ Medical University, Bialystok, Poland
}

Received February 22, 2013

Accepted July 23, 2013

On-line September 10, 2013

\section{Summary}

Diabetes mellitus types 1 and 2 are chronic diseases that cause serious health complications, including dermatologic problems. The diabetic skin is characterized by disturbances in collagen metabolism. A tissue remodeling depends on the degradation of extracellular matrix through the matrix metalloproteinases, which are regulated by e.g. the tissue inhibitors of metalloproteinases. The balance between matrix metalloproteinases (MMPs) and tissue inhibitors of matrix metalloproteinases (TIMPs) is essential to maintain homeostasis in the skin. The aim of this study was to determine the concentration of metalloproteinase 2, tissue inhibitor of metalloproteinase 3 and the concentration of collagen type 1 in unwounded skin of diabetes type 1 and 2 and healthy controls. The treatment of diabetes resulted in a significant decrease of MMP2, increase of TIMP3 and COL1 concentrations in the skin as compared to the untreated diabetic skin. The concentrations of MMP2 in the skin of treated rats did not show significant differences from the healthy control group. TIMP3 concentrations in the skin of treated rats are not returned to the level observed in the control group. Disturbances of the extracellular matrix of the skin are similar in diabetes type 1 and 2. Application of insulin in diabetes therapy more preferably affects the extracellular matrix homeostasis of the skin.

\section{Key words}

Skin homeostasis • Diabetes • Insulin • Metformin

\section{Corresponding author}

M. Knaś, Research Laboratory of Cosmetology, Medical University, 3 Akademicka Str., 15-267 Bialystok, Poland. Fax/phone: +4885745828. E-mail: knass@wp.pl

\section{Introduction}

Diabetes mellitus types 1 and 2 are chronic diseases that cause serious health complications, including not only renal failure and heart disease, but also dermatologic problems. It was shown that skin complications appear during the course of disease, but they may also be the first symptom of diabetes or precede the diagnosis by many years. It is also well-known that the diabetic skin has deficient wound-healing properties and it is characterized by disturbances in collagen metabolism at the site of the unhealing wound. Collagen, mainly collagen type 1 , is a major component of the skin and plays an important role in the healing and remodeling of the skin structure (Bermudez et al. 2011). Tissue remodeling depends on the degradation of extracellular matrix through the action of the matrix metalloproteinases (MMPs), a family of zinc-dependent endopeptidases (Nagase et al. 2006). During healing, MMPs play an important function and it was proven that the abnormal expression could be a reason of impaired proper function and wound healing (Wall et al. 2003, Tong et al. 2012). Their upregulation facilitates aging and cancer (Philips et al. 2011). The activities of most MMPs are very low in the normal skin, but their expression is controlled by the inflammatory cytokines, hormones and growth factors. MMPs activities are regulated by the activation of the precursor zymogens and inhibition by endogenous inhibitors, tissue inhibitors of matrix metalloproteinases (TIMPs) (Page-McCaw et al. 2007). The balance between MMPs and TIMPs is essential to 
maintain homeostasis in the skin, with a MMPs/TIMPs imbalance associated with breakdown of the extracellular space in the skin. It was shown that this imbalance may reflect a disordered microenvironment due to uncontrolled hyperglycemia.

There is little known about the remodeling of the extracellular matrix of the skin in unwounded surfaces during the course and treatment of different types of diabetes (Bermudez et al. 2011). The aim of this study was to determine the concentration of matrix metalloproteinase 2 (MMP2) and tissue inhibitor of matrix metalloproteinase 3 (TIMP3) and concentration collagen type 1 (COL1) in unwounded skin of diabetes type 1 (streptozotocin-induced; STZ-induced) and type 2 (diet-induced) and healthy control rats. The aim of this study was also to assess the impact of diabetes types 1 and 2 on the metabolism of the extracellular matrix of the skin.

\section{Materials and Methods}

\section{Experimental animals}

Healthy adult male Wistar rats were obtained from experimental animal house, Department of Experimental Pharmacology, Medical University in Bialystok. The rats were divided randomly into seven groups of 8 rats each. Each rat weighed between $160-180 \mathrm{~g}$ and was housed separately in standard conditions $\left(20-21^{\circ} \mathrm{C} \pm 2{ }^{\circ} \mathrm{C}, 12 \mathrm{~h}\right.$ of light/12 h of dark). They had unlimited access to water, and they were fed $a d$ libitum on a standard laboratory rat chow (Agropol, Motycz, Poland). The study was approved by the Local Committee on the Ethical Use of Animals Medical University in Bialystok, Poland (N. 113/275/81 P).

Control rats $(\mathrm{C})$ were injected intraperitoneally (i.p.) with $0.9 \% \mathrm{NaCl}$; the mean glycemia at the end of the experiment $-92.5 \pm 7.21 \mathrm{mg} / \mathrm{dl}$.

\section{Diabetic animals}

Diabetes type 1: after overnight fasting, diabetes mellitus was induced in two groups (untreated diabetes type 1 (D1), insulin treated diabetes type $1(\mathrm{D} 1+\mathrm{I})$ by a single injection of STZ (Sigma, USA; $50 \mathrm{mg} / \mathrm{kg}$, i.p. prepared in the citrate buffer $(0.1 \mathrm{M}, \mathrm{pH} 4.5)$ (Al-Bayaty and Abdulla 2012). Blood was drawn from the tail on the seventh day after STZ injection. The blood glucose level was used to confirm the development of diabetes$367.45 \pm 15.64 \mathrm{mg} / \mathrm{dl}$. Treatment of diabetes was started in one group $(\mathrm{D} 1+\mathrm{I})$ during the fifth week after STZ injection. The rats were treated with insulin (6-8 U s.c., depending on blood glucose level) once a day for 3 weeks; the mean glycemia at the end of the experiment $-98.6 \pm 5.34 \mathrm{mg} / \mathrm{dl}$.

Diabetes type 2: after overnight fasting, diabetes mellitus was induced in two groups (untreated diabetes type 2 (D2), metformin treated diabetes type $2(\mathrm{D} 2+\mathrm{M})$ by a high-fat-diet (HFD) (Hou et al. 2012) with $60 \%$ fat, $20 \%$ protein, $20 \%$ carbohydrate. The blood glucose level was used to confirm the development of diabetes $349.11 \pm 17.24 \mathrm{mg} / \mathrm{dl}$. Treatment of diabetes was started in one group (D2+M) during the fifth week after the highfat diabetes-inducing diet. The rats were treated with metformin (300-500 mg/kg (per os), depending on blood glucose level) once a day for 3 weeks; the mean glycemia at the end of the experiment $-99.2 \pm 4.11 \mathrm{mg} / \mathrm{dl}$.

To assess the effect of metformin and insulin on the homeostasis of the skin matrix drugs were administered to healthy rats ( $\mathrm{I}$ and $\mathrm{M}$ ) for 3 weeks at a dose of the non-hypoglycemic effect ( $1 \mathrm{U}$ of insulin s.c. once a day, $300 \mathrm{mg}$ of metformin per os once a day). A mean glycemia at the end of the experiment in both groups was similar (I - 93.8 $\pm 2.68 \mathrm{mg} / \mathrm{dl}, \mathrm{M}-92.9 \pm 4.17$ $\mathrm{mg} / \mathrm{dl})$.

The animals were anesthetized by i.p. injection of pentobarbital in a dose of $80 \mathrm{mg} / \mathrm{kg}$ body weight. Skin samples were taken from a shaved dorsal neck of each rat (approximately $2 \mathrm{~cm}^{2}$ ). One part of the skin was immediately frozen in liquid nitrogen and stored at $80^{\circ} \mathrm{C}$ until use, the second was fixed in $10 \%$ neutral formaldehyde for histological analysis.

The tail blood was drawn on the seventh day after the HFD, and the blood glucose levels were estimated using a glucometer (Accu-Check Active, Roche, France).

\section{Histological evaluation of the unwounded skin}

Paraffin-embedded skin samples were cut into $5 \mu \mathrm{m}$-thick slices, and the cutaneous histological characteristics were observed under a light microscope Opta-tech MN800 (Opta-tech, Poland) (200x) after hematoxylin and eosin staining. For quantitative analysis the pixel intensity of the collagen was determined with MultiScan v.8.08 (Computer Scanning System, Poland).

\section{Homogenization}

All the animals used for experiments were asleep under anesthesia and sacrificed. Skin sample were weighted before homogenization and then rinsed in ice- 
cold PBS (0.02 mol/1, pH 7.0-7.2). Minced the tissues to small pieces and homogenized 6 times diluted in PBS with a glass-homogenizer (Omni TH, Omni International, USA) on ice. The resulting suspension was sonicated with an ultrasonic cell disrupter (UP 400S, Hielscher, Germany; $1800 \mathrm{~J}$ per sample, $20 \mathrm{~s} \mathrm{x} 3$ on ice to further break the cell membranes. After that, the homogenates were centrifuged for $5 \mathrm{~min}, 4^{\circ} \mathrm{C}, 5000 \times \mathrm{g}$ (MPW 351, Poland) to remove the supernatant and assayed immediately.

\section{Assays}

The concentrations of matrix metalloproteinase 2 (MMP2) and tissue inhibitor of matrix metalloproteinase 3 (TIMP3) and concentration collagen type 1 (COL1) were determined by ELISA (USCN, Life Science, China) according to manufactured. Protein content was determined by bicinchoninic acid BCA method with bovine albumin as a standard (PIERCE BCA Protein Assay Kit). All analyses were performed in duplicate.

\section{Statistics}

Results were reported as median, minimum and maximum. Statistical analysis was performed using Statistica 10.0 (Statsoft, Cracow, Poland). Kruskal-Wallis ANOVA test was used to study the significant differences between groups. Spearman Correlation Coefficient was used to study the associations between the variables. The statistical significance was defined as $\mathrm{p} \leq 0.05$.

\section{Results}

Mean glycemia of rats from D1 and D2 groups was significantly higher $(\mathrm{p}<0.01, \mathrm{p}<0.01)$ compared to the control healthy group $(\mathrm{C})$, and to the group treatment by insulin $(\mathrm{D} 1+\mathrm{I})$ and metformin $(\mathrm{D} 2+\mathrm{M}) \quad(\mathrm{p}<0.01$, $\mathrm{p}<0.01$, respectively). After application of insulin and metformin, blood glucose normalization was obtained to the level obtained in the group of healthy rats $(p=0.464$, $\mathrm{p}=0.517$, respectively).

\section{Histological examination}

In the skin section stained with hematoxylin and eosin, we observed the cellular and tissue patterns, which allow as to recognize the tissue and to establish the diagnosis based on morphological parameters. For quantitative analysis points (pixels) were selected in regions with collagen fibers and the area without tissue (control) for determining the intensity. The pixel intensity of collagen in the skin (Table 1) of untreated diabetic D1 and $\mathrm{D} 2$ rats were significantly lower in comparison to the control ( $p<0.01$, respectively). In the skin of diabetic D1 rats treated with insulin $(\mathrm{D} 1+\mathrm{I})$ the pixel intensity of collagen were significantly higher in comparison to the untreated D1 diabetic group $(p=0.02)$. In the skin of healthy rats substituted with insulin (I) the pixel intensity of collagen were significantly higher in comparison to the control ( $p=0.01)$. However, the pixel intensity of collagen in the skin of diabetic rats treated with metformin $(\mathrm{D} 2+\mathrm{M})$ and in the skin of healthy rats substituted with metformin (M) were at the similar level as in the control.

Table 1. Analysis of the pixel intensity of collagen 1 after hematoxylin and eosin staining.

\begin{tabular}{lc} 
Group & $\begin{array}{c}\text { The pixel intensity of collagen } \\
\text { (median, min-max) }\end{array}$ \\
\hline$C$ & $43.41(40.25-46.74)$ \\
$D 1$ & $26.97(24.56-29.11)$ \\
$D 1+I$ & $89.12(79.96-91.28)$ \\
$I$ & $92.55(88.03-100.01)$ \\
$D 2$ & $24.81(18.79-28.74)$ \\
$D 2+M$ & $41.26(37.63-45.08)$ \\
$M$ & $42.09(39.03-46.11)$ \\
\hline
\end{tabular}

Histological examination analysis of the skin from untreated diabetic type 1 (D1) and 2 (D2) rats showed many skin lesions, such as a decrease in skin thickness, disappearance of the multilayer epithelial structure of the epidermis, atrophy and degeneration of collagen fibers in the dermis in comparison to the healthy control (Fig. 1a). Application of insulin for the treatment of diabetes resulted in a marked change of the construction of all skin layers of the tested animals. Thickening of the epidermis, the stratum corneum, in particular of the granular layer, was observed. Thickening of the dermis and the more compact arrangement of the dermal collagen fibers were observed, both in comparison to the group of diabetic rats as well as the control healthy group. Histological analysis of the diabetic skin rats treated with metformin showed that this form of therapy improves the collagen fibers of the dermis, but does not improve the construction of the epidermis (Fig. 1f). In the healthy group administered with insulin (I) (Fig. 1d), changes were as shown in the group D1+I (Fig. 1c), but with a higher intensity. The material collected from 
healthy rats treated with metformin did not differ from the control group of healthy rats (C) (Fig. 1g).

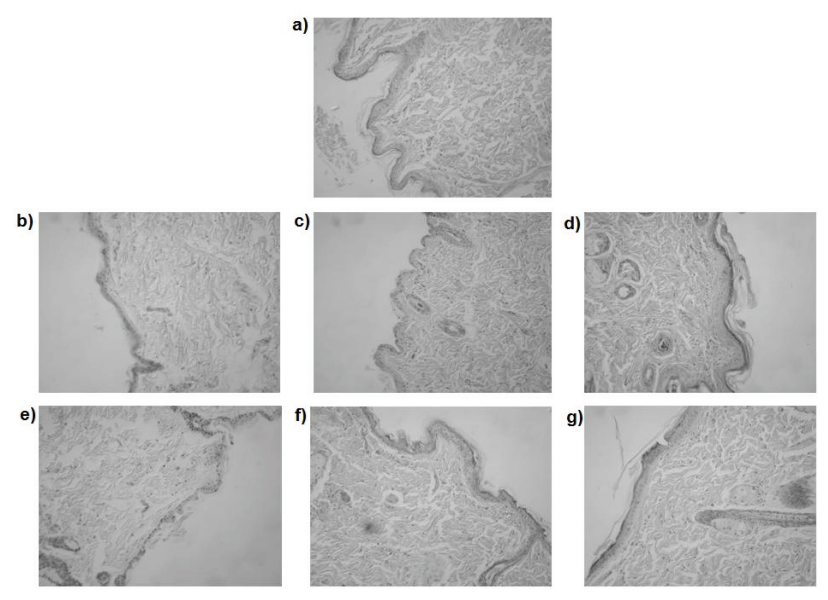

Fig. 1. Histological feature of the skin from control rats (a), untreated diabetic type 1 (b) and 2 (e), diabetic rats treated with insulin (c) and metformin (f), healthy rats administered with insulin (d) and metformin (g). The histological characteristics were observed under a light microscope (200x) after hematoxylin and eosin staining.

\section{Concentration of $M M P 2$}

MMP2 concentrations in the skin of untreated diabetic D1 and D2 rats were significantly higher in comparison to the MMP2 concentration of the control $(\mathrm{p}<0.01)$ and diabetic rats treated with insulin and metformin ( $\mathrm{p}<0.01$, in both). The concentrations of MMP2 in the skin of diabetic rats treated with insulin and metformin and also in healthy rats substituted with insulin and metformin were similar as in the healthy control rats $(\mathrm{p}=0.24, \mathrm{p}=0.4, \mathrm{p}=0.9, \mathrm{p}=0.6$, respectively) (Fig. 2a).

\section{Concentration of TIMP3}

The highest concentration of TIMP 3 was noted in the control healthy rats in comparison to the other groups, but significantly only so for D1 and D2 ( $<<0.01$, in both). The treatment with insulin (D1+I) as well as metformin $(\mathrm{D} 2+\mathrm{M})$ resulted in increased concentrations of TIMP3 in the rat skin as compared to the untreated D1 and D2 diabetic groups ( $p<0.01, p=0.03$, respectively) (Fig. $2 b$ ).

\section{Ratio of MMP2/TIMP3}

Ratios of MMP2/TIMP3 in the untreated diabetic D1 and D2 groups were significantly higher in comparison to the healthy control $(\mathrm{p}<0.01$, in both) and diabetic rats treated with insulin $(\mathrm{D} 1+\mathrm{I})(\mathrm{p}<0.01)$ and metformin $(\mathrm{D} 2+\mathrm{M})(\mathrm{p}<0.01)$. Ratios of MMP2/TIMP3 in the skin of insulin-treated diabetic (D1+I) and healthy rats substituted with insulin (I) were the same as in the skin of the control healthy rats $(C)(p<0.32, p<0.29)$ however, ratios in the skin of diabetic rats treated with metformin $(\mathrm{D} 2+\mathrm{M})$ and healthy rats substituted with metformin $(\mathrm{M})$ were higher in comparison to the control $(\mathrm{p}<0.01 ; \mathrm{p}<0.01$, respectively) (Fig. 3).

\section{Concentration of collagen 1}

STZ-induced diabetes (D1) caused significantly decreased COL1 concentrations in the skin in comparison to the healthy control rats (C) $(\mathrm{p}<0.01)$, whereas treatment with insulin (D1+I) caused significant increases in COL1 concentrations in comparison to the diabetic untreated (D1) $(\mathrm{p}<0.01)$ and healthy control rats $(\mathrm{C})$ $(\mathrm{p}<0.01)$. COL1 concentrations in the skin of rats treated with metformin $(\mathrm{D} 2+\mathrm{M})$ were at a similar level as in the control healthy rats (C) $(\mathrm{p}=0.82)$, although it was significantly higher than in the skin of untreated diabetic rats (D2) $(p<0.01)$. COL1 concentrations in the skin of rats substituted with insulin (I) were significantly higher $(\mathrm{p}=0.002)$, but in the skin of rats substituted with metformin (M), they were similar $(\mathrm{p}=0.76)$ to those in the control healthy rats (C) (Fig. 4).

There was a very strong correlation between concentrations of MMP2 and TIMP3 in D1 and $\mathrm{D} 2$ groups $(\mathrm{p}=0.049, \mathrm{r}=-0.76 ; \mathrm{p}=0.047, \mathrm{r}=-0.73$, respectively), as well as between the concentrations of MMP2 and COL1 in D1 and D2 groups $(p<0.01$, $\mathrm{r}=-0.99 ; \mathrm{p}=0.003, \mathrm{r}=-0.85$ ).

\section{Discussion}

Reconstruction of the skin extracellular matrix is a physiological occurrence that occurs continuously, runs in cooperation with a number of enzymes from cells present in the skin, as well as migrant cells, i.e. neutrophils, monocytes, mast cells and macrophages. As a result, the interaction of the cells occurs in the controlled synthesis of enzymes and their activators, inhibitors, and growth factors. This provides a balance between the synthesis and degradation of extracellular matrix components, which determines the integrity and proper function of the skin. The key enzymes involved in the degradation of the skin extracellular matrix, including collagen 1, are matrix metalloproteinase and their tissue inhibitors (Bermudez et al. 2011).

Skin complications during diabetes mellitus are a frequent clinical problem. Skin lesions are more common in type 2 than type 1 diabetes mellitus - 

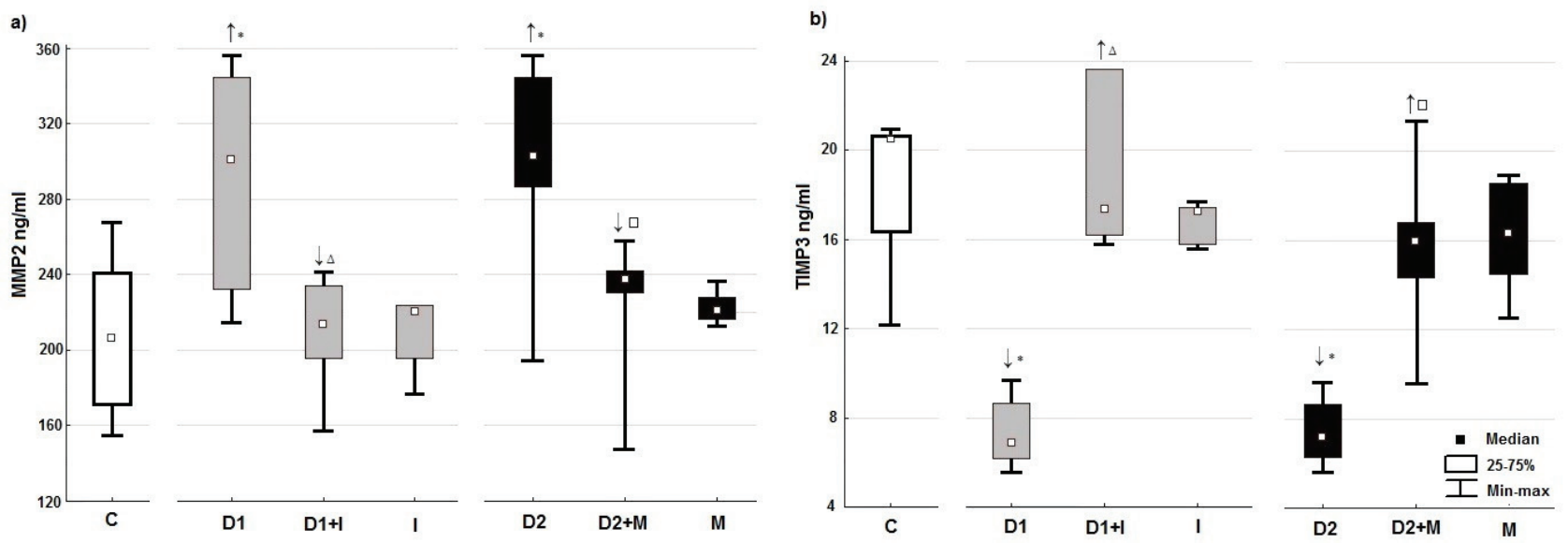

Fig. 2. MMP2 and TIMP3 concentrations $(\mathrm{ng} / \mathrm{ml})$ in the skin of control, untreated diabetic D1 and D2 rats, diabetic rats treated with insulin and metformin and healthy rats substituted with insulin and metformin. Abbreviations: $\uparrow-$ increase, $\downarrow-$ decrease, $*-p<0.01$ in comparison to control group, $\Delta-p<0.001$ in comparison to untreated diabetic type 1 rats, $\square-p<0.01$ in comparison to untreated diabetic type 2 rats.

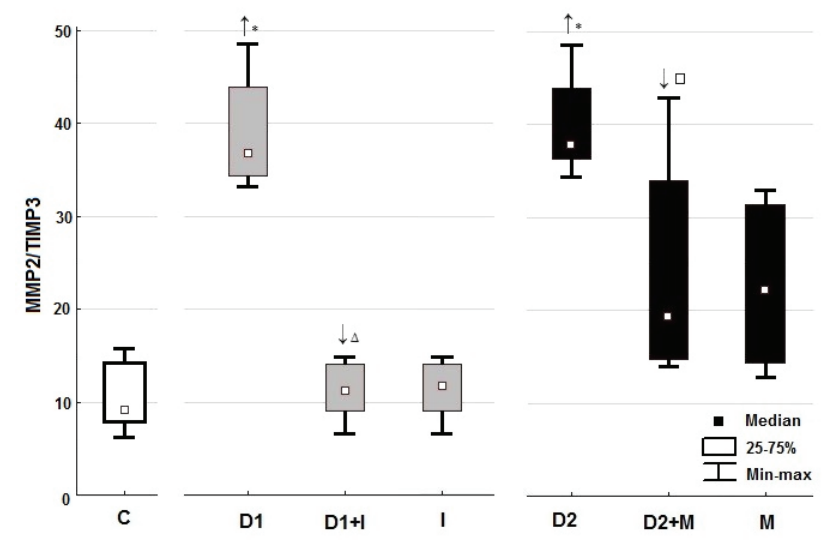

Fig. 3. Ratio of MMP2/TIMP3 in the skin of control, untreated diabetic D1 and D2 rats, diabetic rats treated with insulin and metformin and healthy rats substituted with insulin and metformin. Abbreviations: $\uparrow-$ increase, $\downarrow-$ decrease, $*-p<0.01$ in comparison to control group, $\Delta-p<0.01$ in comparison to untreated diabetic type 1 rats, $\square-p<0.01$ in comparison to untreated diabetic type 2 rats.

however, the reasons for this incidence are unknown (van Hattem et al. 2008). The majority of studies, that have investigated the remodeling of the diabetic skin, have examined it in the wounded state, not at the baseline. It was shown that the normal process of wound healing is disrupted and a chronic wound may results. One of the key factors, in addition to impaired blood supply, resulting in the development of non-healing wounds is the impaired expression and the relationship between matrix metalloproteinase and their tissue inhibitors, which consequently interferes with the metabolism of collagen.

In the present literature, there is a lack of information on the metabolism of the extracellular matrix
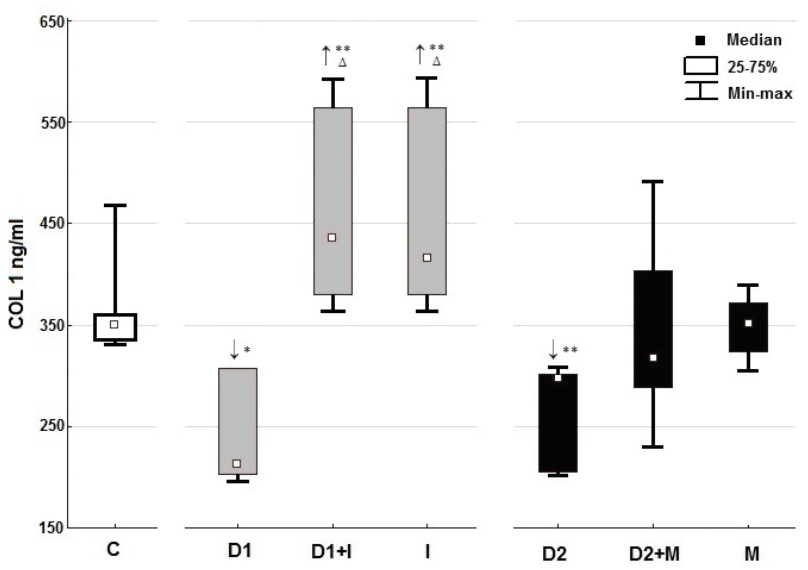

Fig. 4. COL1 concentrations $(\mathrm{ng} / \mathrm{ml})$ in the skin of control, untreated diabetic D1 and D2 rats, diabetic rats treated with insulin and metformin and healthy rats substituted with insulin and metformin. Abbreviations: $\uparrow$ - increase, $\downarrow$ - decrease, $*$ - $\mathrm{p}<0.01$ in comparison to control group, $* *-p<0.01$ in comparison to control group, $\Delta-p<0.01$ in comparison to untreated diabetic type 1 rats.

of the skin without any macroscopic lesions in diabetes types 1 and 2. The effect of the primary treatment for both diabetes types of skin enzymatic activity is also unknown. In our experimental model, we wanted to assess the impact of diabetes types 1 and 2 to change the function of enzymes involved in the remodeling of the connective tissue of the dermis, which is characterized by the greatest content of collagen type 1 . The most of the publications describe interdependencies between MMP-2 and TIMP-2. It is known that TIMP-1 inhibits the activity of most MMPs, with the exception of MT1-MMP and MMP-2, TIMP-2 inhibits the activity of most MMPs, except MMP-9 and also to the latent form pro MMP-2 (Folgueras et al. 2004, Amalinei et al. 2007). TIMP-3 
inhibits the activity of MMP-1, MMP-2, MMP-3, MMP-9 and MMP-13. TIMP-3 may bind to sulfated glycosaminoglycans of the extracellular matrix and then inhibits MMP-3 (Seo et al. 2003, Collette et al. 2004, Amalinei et al. 2007). On this basis, we investigated the mutual relationship between MMP-2 and TIMP-3. Therefore, our choice fell on MMP2, since it is involved in the breakdown of COL1 (Jinnin 2010), as well as the observed elevated levels during tissue angiogenesis in both physiological and impaired statuses during diabetes (Dalton et al. 2005).

Our results indicate that homeostasis disturbances of the skin extracellular matrix in diabetes types 1 and 2 do not differ statistically. We showed that the concentrations of MMP2 increase during diabetes in comparison to the healthy control. We also observed significant decreases of type 1 collagen and TIMP3 concentrations and the significantly higher ratio of the MMP2/TIMP3. The correlation between the concentrations of MMP2 and TIMP3 indicates a very strong influence of hyperglycemia on the changes in these enzyme levels. The correlation between MMP2 and COL1 may prove the mutual unconditional relationship of the amount of MMP2 and COL1 in the diabetic skin. The observed changes may demonstrate developing angiogenic processes that compensate for the destruction of the blood vessels, resulting from the structural protein glycosylation in diabetes (Dalton et al. 2005). In contrast the elevated ratio of the MMP2/TIMP3 in the diabetes skin suggests an imbalance between the degradation and the synthesis of extracellular matrix, as evidenced by the significantly decreased collagen 1 concentrations. The skin blood flow disturbances in diabetes, therefore diminish the ability to remove the degradation products of collagen, may cause skin lesions that are characteristic of chronic diabetes. On the other hand, in the presence of hyperglycemic, glucose molecules react with the lysine and hydroxylysine $\varepsilon$ - amidocyanogen of skin collagen. This leads to non-specific glycosylation of collagen with subsequent formation of advanced glycation end products (AGE), thereby disrupting the metabolism of collagen. AGEs bind to macrophages and other cells through the AGE receptors, which may cause damage to the skin (Chen et al. 2009). This process may reduce the proper concentration of collagen. Another explanation for the phenomena occurring can be that glycosylation changes the quaternary structure of collagen, which is not recognized by the antibodies used in the assay. It has been shown that excessive glycosylation of collagen damages the skin structure, which was observed in the histological examination as many skin changes: a decrease in skin thickness, disappearance of the multilayer epithelial structure of the epidermis, atrophy and degeneration of collagen fibers in the dermis in comparison to the control rats (Chen et al. 2009). The treatment of diabetes, regardless of the selection of the therapeutic method $(\mathrm{D} 1+\mathrm{I}$ and $\mathrm{D} 2+\mathrm{M})$, resulted in a significant decrease of MMP2, increase of TIMP3 and COL1 concentrations in the skin as compared to the groups of untreated diabetic rats (D1 and D2). Moreover, the concentrations of MMP2 in the skin of treated rats $(\mathrm{D} 1+\mathrm{I}$ and $\mathrm{D} 2+\mathrm{M})$ did not show significant differences from the healthy control group (C). TIMP3 concentrations in the skin of treated rats $(\mathrm{D} 1+\mathrm{I}$ and $\mathrm{D} 2+\mathrm{M})$ are not returned to the level observed in the control group (C). This may be due to the increased susceptibility to glycosylation and degradation, or a feedback promotes angiogenesis. Insulin treatment (D1+I) results in a significant increase in the concentrations of the skin collagen 1 in comparison to the healthy rats $(\mathrm{C})$, which probably due to its hypoglycemic and anabolic activities. It has been shown that insulin increases amino acid uptake in cells and enhances protein synthesis, stimulates RNA synthesis and formation of polypeptides during the process of translation (Miers and Barrett 1998, Sunahara et al. 2012). Under the anabolic action on proteins, insulin behaves like a growth hormone increases the mRNA synthesis and DNA transcription, thus increasing the protein mass in the body (Yeap et al. 2013). The results obtained in a group of healthy rats treated with insulin (I), confirm that the anabolic mechanism of insulin determines the COL1 concentrations in the skin. As a result, metformin sensitizes peripheral cells to the hypoglycemic insulin, but has no anabolic effect on the protein (Boyle et al. 2010, Elgebaly et al. 2010). The subliminal nonhypoglycemic metformin dose applied to healthy rats (M) did not change the extracellular matrix homeostasis of the skin. Equalization of COL1 concentrations in the diabetic group treated with metformin $(\mathrm{D} 2+\mathrm{M})$ is probably the result of the glycemic control and inhibits the formation of advanced glycation end products (AGE), which restores the physiological metabolism of COL1. The above-described changes in the concentration of COL1 were also observed in the histological studies of the skin (Fig. 1c,f).

Both MMP2 and TIMP3 influence directly or indirectly the homeostasis of collagen 1 in the skin, and 
play an important role in tissue remodeling. As mentioned earlier, the MMP2/TIMP3 ratio is very important to maintain healthy skin. The MMP2/TIMP3 ratio in the skin of treated rats $(\mathrm{D} 1+\mathrm{I}$ and $\mathrm{D} 2+\mathrm{M})$ is reduced significantly as compared to the diabetic rats (D1 and D2). However, only the MMP2/TIMP3 ratio in the group of rats treated with metformin $(\mathrm{D} 2+\mathrm{M})$ is significantly higher than in healthy controls (C). Only the insulin treatment $(\mathrm{D} 1+\mathrm{I})$ returns the MMP2/TIMP3 ratio to the level observed in the healthy controls $(\mathrm{C})$.

This result may suggest that destruction of collagen and disorders of the skin remodeling mechanisms in the insulin-treated group $(\mathrm{D} 1+\mathrm{I})$ is arrested at least after 4 weeks of treatment.

Relative misalignment between MMP2 and
TIMP3 after treatment with metformin suggests an imbalance between the degradation and the synthesis of extracellular matrix in the group of animals treated by the appropriate type of treatment (Zhang and Bi 2011). This process may be responsible for the high prevalence of skin diseases in type 2 diabetes, in which metformin is the first-choice oral drug in the treatment.

\section{Conflict of Interest}

There is no conflict of interest.

\section{Acknowledgements}

This work was supported by the Medical University of Białystok Grant No. 113/275/81 P.

\section{References}

AL-BAYATY F, ABDULLA MA: A comparison of wound healing rate folowing treatment with aftamed and chlorine dioxide gels in streptozotocin-induced diabetic rats. Evid Based Complement Alternat Med 2012: doi: $10.1155 / 2012 / 468764$.

AMALINEI C, CARUNTU ID, BALAN RA: Biology of metalloproteinases. Rom J Morphol Embryol 48: 323-334, 2007.

BERMUDEZ DM, HERDRICH BJ, XU J, LIND R, BEASON DP, MITCHELL ME, SOSLOWSKY LJ, LIECHTY KW: Impaired biochemical properties of diabetic skin. Implications in pathogenesis of diabetic wound complications. Am J Pathol 178: 2215-2223, 2011.

BOYLE JG, MCKAY GA, FISHER M: Drugs for diabetes: part 1 metformin. Br J Cardiol 17: 231-234, 2010.

CHEN X, LIN W, LU S, SHI Y, ZOU J, LIU Z, LIAO W: Insulin prevents latent skin lesions by inhibiting the generation of advanced glycation end products in Streptozotocin-induced diabetes rats. Endocr Pathol 20: 163-169, 2009.

COLletTE T, BELlEHUMEUR C, KATS R, MAHEUX R, MAILlOUX J, VILlENEUVE M, AKOUM A: Evidence for an increased release of proteolytic activity by the eutopic endometrial tissue in women with endometriosis and for involvement of matrix metalloproteinase-9. Hum Reprod 19: 1257-1264, 2004.

DALTON SJ, MITCHELL CD, WHITING CV, TARLTON JF: Abnormal extracellular matrix metabolism in chronically ischemic skin: a mechanism for dermal failure in leg ulcers. J Invest Dermatol 125: 373-379, 2005.

ELGEBALY MM, PRAKASH R, LI W, OGBI S, JOHNSON MH, MEZZETTI EM, FAGAN SC, ERGUL A: Vascular protection in diabetic stroke: role of matrix metalloprotease-dependent vascular remodeling. J Cereb Blood Flow Metab 30: 1928-1938, 2010.

FOLGUERAS AR, PENDÁS AM, SÁNCHEZ LM, LÓPEZ-OTÍN C: Matrix metalloproteinases in cancer: from new functions to improved inhibition strategies. Int J Dev Biol 48: 411-424, 2004.

HOU L, LIAN K, YAO M, SHI Y, LU X, FANG L, HE T, JIANG L: Reduction of n-3 PUFAs, specifically DHA and EPA, and enhancement of peroxisomal beta-oxidation in type 2 diabetic rat heart. Cardiovasc Diabetol 2012: doi: 10.1186/1475-2840-11-126.

JINNIN M: Mechanisms of skin fibrosis in systemic sclerosis. J Dermatol 37: 11-25, 2010.

MIERS WR, BARRETT EJ: The role of insulin and other hormones in the regulation of amino acid and protein metabolism in humans. J Basic Clin Physiol Pharmacol 9: 235-253, 1998.

NAGASE H, VISSE R, MURPHY G: Structure and function of matrix metalloproteinases and TIMPs. Cardiovasc Res 69: 562-573, 2006. 
PAGE-MCCAW A, EWARD AJ, WERB Z: Matrix metalloproteinases and the regulation of tissue remodeling. Nat Rev Mol Cell Biol 8: 221-233, 2007.

PHILIPS N, AULER S, HUGO R, GONZALEZ S: Benefical regulation of matrix metalloproteinases for skin health. Enzyme Res 2011: doi: 10.4061/2011/427285.

SEO DW, LI H, GUEDEZ L, WINGFIELD PT, DIAZ T, SALLOUM R, WEI BY, STETLER-STEVENSON WG: TIMP-2 mediated inhibition of angiogenesis. Cell 114: 171-180, 2003.

SUNAHARA KKS, SANNOMYA P, MARTINS JO: Briefs on insulin and innate immune response. Cell Physiol Biochem 29: 1-8, 2012.

TONG M, TUK B, SHANG P, HEKKING IM, FIJNEMAN EM, GUIJT M, HOVIUS SE, VAN NECK JW: Diabetesimpaired wound healing is improved by matrix therapy with heparan sulfate glycosaminoglycan mimetic OTR4120 in rats. Diabetes 61: 2633-2641, 2012.

VAN HATTEM S, BOOTSMA AH, THIO HB: Skin manifestations of diabetes. Clev Clin J Med 75: 772, 774, 776-777, 2008.

WALL SJ, SAMPSON MJ, LEVELL N, MURPHY G: Elevated matrix metalloproteinase-2 and -3 production from human diabetic dermal fibroblasts. Br J Dermatol 149: 13-16, 2003.

YEAP BB, CHUBB PSA, LOPEZ D, HO KK, HANKEY GJ, FLICKER L: Associations of insulin-like growth factor-I and its binding proteins, and testosterone, with frailty in older men. Clin Endocrinol (Oxf) 78: 752-759, 2013.

ZHANG LX, BI LJ: Alterations of collagen-I, MMP-1 and TIMP-1 in the periodontal ligament of diabetic rats under mechanical stress. J Periodont Res 46: 448-455, 2011. 